\title{
Análisis de los resultados del programa de ecocardiografía funcional neonatal en un hospital pediátrico de tercer nivel
}

\author{
Daniel Ibarra-Ríos ${ }^{1}$, Horacio Márquez-González ${ }^{2 *}$, Alejandra Quiroga-Valdés ${ }^{1}$, Adrián E. Guzmán-Arce ${ }^{3}$, \\ Dina Villanueva-García ${ }^{1}$ y Raúl Villegas-Silva ${ }^{1}$ \\ ${ }^{1}$ Departamento de Neonatología; ${ }^{2}$ Departamento de Investigación Clínica. Hospital Infantil de México Federico Gómez, Ciudad de México, México; \\ ${ }^{3}$ Departamento de Neonatología, Hospital de Niños Roberto Gilbert E, Guayaquil, Ecuador
}

\begin{abstract}
Resumen
Introducción: Se ha demostrado la utilidad del estudio ecocardiográfico en las enfermedades que afectan a los neonatos en estado crítico, por lo que se debe capacitar al neonatólogo para la toma y la interpretación de variables hemodinámicas que apoyen la toma de decisiones. El objetivo de este estudio fue describir los resultados del programa de ecocardiografía funcional neonatal y comparar las variables clínicas y hemodinámicas entre sobrevivientes y no sobrevivientes. Métodos: Durante un periodo de 2 años se realizó un estudio observacional, transversal y comparativo en neonatos que fueron evaluados con ecocardiografía funcional neonatal por alteraciones hemodinámicas del conducto arterioso persistente, hipertensión pulmonar aguda y crónica, estado de choque y búsqueda de trombos y vegetaciones. Se evaluaron parámetros de funcionalidad de los ventrículos derecho e izquierdo, presión pulmonar y subrogados de sobrecarga pulmonar. Se realizó un análisis comparativo ( $U$ de Mann Whitney y $\chi^{2}$ ). De acuerdo con los resultados, se emitieron recomendaciones para el inicio, el ajuste o el retiro de fármacos vasoactivos. Resultados: Se realizaron 269 estudios en 119 neonatos $(64.7 \%$ prematuros, mortalidad del 15\%) atendidos por hipertensión pulmonar aguda (38\%), conducto arterioso persistente (27\%), choque (19\%), hipertensión pulmonar crónica (14\%) o búsqueda de trombos (2\%). Se recomendó cambiar el manejo en el $45 \%$ de los estudios. El grupo de no sobrevivientes presentó diferencias significativas en los parámetros de función sistólica del ventrículo derecho y aumento de su poscarga. Conclusiones: La causa principal de la evaluación con ecocardiografía funcional neonatal fue la hipertensión pulmonar. De forma global, se recomendó un ajuste farmacológico en el $45 \%$ de los casos.
\end{abstract}

Palabras clave: Choque. Ecocardiografía. Hipertensión pulmonar. México. Persistencia del conducto arterioso. Recién nacido.

\section{Analysis of the results of the neonatal functional echocardiography program in a third-level pediatric hospital}

\section{Abstract}

Background: Echocardiography is useful in the group of comorbidities of critically ill newborns. The targeted neonatal echocardiography program trains neonatologists for acquiring and interpreting hemodynamic variables to support decision making. This study aimed to describe the results of the functional echocardiography program (fNE) and compare clinical and hemodynamic variables between survivors and non-survivors. Methods: Observational, cross-sectional, and comparative

Correspondencia:

*Horacio Márquez-González

E-mail: horaciohimfg@gmail.com
Fecha de recepción: 12-02-2020

Fecha de aceptación: 16-05-2020 DOI: 10.24875/BMHIM.20000036
Disponible en internet: 17-07-2020 Bol Med Hosp Infant Mex. 2020;77(4):178-185 www.bmhim.com 1665-1146/@ 2020 Hospital Infantil de México Federico Gómez. Publicado por Permanyer. Este es un artículo open access bajo la licencia CC BY-NC-ND (http://creativecommons.org/licenses/by-nc-nd/4.0/). 
study of neonates that received a fNE evaluation for hemodynamic disturbances related to patent ductus arteriosus (PDA), acute and chronic pulmonary hypertension $(\mathrm{aPH}, \mathrm{cPH})$, state of shock and thrombus/vegetations surveillance for two years. Functional parameters of the right and left ventricle, pulmonary pressure, and surrogates of pulmonary over circulation were assessed. Comparative analysis with $U$ Mann Whitney test and $\chi^{2}$ was performed. Based on the results, recommendations to start, adjust, or withdraw vasoactive medications were issued. Results: Of 269 studies on 119 neonates (65\% premature, $15 \%$ mortality), the reasons for consultation were aPH (38\%), PDA (27\%), shock (19\%), cPH (14\%), and thrombus surveillance $(2 \%)$. A change in management was recommended on $45 \%$ of studies. Non-survivors presented significant differences in the right ventricular (RV) systolic performance and an increased right ventricular afterload. Conclusions: The main indication for fNE was pulmonary hypertension. A pharmacological adjustment was recommended on $45 \%$ of the cases.

Key words: Shock. Echocardiography. Pulmonary hypertension. Mexico. Patent ductus arteriosus. Newborn.

\section{Introducción}

Se calcula que 88 de cada 1000 recién nacidos vivos en el mundo, de los cuales la mitad son prematuros con un peso $<900 \mathrm{~g}$, requieren atención por alguna causa en una unidad de cuidados intensivos neonatales (UCIN) ${ }^{1}$. En la UCIN, la toma de decisiones se basa en la historia clínica y en el monitoreo continuo de los signos vitales (presión arterial, frecuencia cardiaca, saturación periférica, llenado capilar, diuresis) y del equilibrio ácido-base. En la actualidad, existe una creciente necesidad de complementar estas evaluaciones con estudios no invasivos y precisos que puedan realizarse en la cama del paciente. Tal es el caso de las acciones tomadas por los anestesiólogos, los intensivistas y los médicos de urgencias que emplean el ultrasonido en su práctica diaria ${ }^{2,3}$. En particular, los neonatos en cuidados críticos requieren asistencia mecánica ventilatoria, además de ser susceptibles a los cambios de temperatura y movilización, por lo que la realización de estudios de gabinete con fines diagnósticos puede representar un riesgo mayor que el posible beneficio. Por estas razones, se ha extendido la experiencia de otros cursos validados con estos mismos fines al contexto de la UCIN y se ha creado el programa focalizado para el neonatólogo, que adiestra para la realización y la interpretación de mediciones ecocardiográficas que evalúan parámetros hemodinámicos. Dichos programas académicos se encuentran acreditados en diversos países (Estados Unidos, Canadá, España, Australia). En Norteamérica ${ }^{4}$ y Europa $^{5}$ se han publicado guías para aplicar estos conocimientos. Para los propósitos de este reporte, se utilizará el término "programa de ecocardiografía funcional neonatal $(\mathrm{EfN}){ }^{\circ}$. En México, algunos centros hospitalarios de tercer nivel han dado prioridad a la necesidad de incorporar este tipo de prácticas y han enviado a los neonatólogos a capacitarse al extranjero. En el instituto sede de este estudio, el programa EfN se incorporó en 2017, con la dinámica aprendida en el centro formador: a partir de una consulta hemodinámica, se integran los hallazgos de EfN y se emite una recomendación?.

El objetivo del estudio fue describir los resultados del programa EfN y comparar las variables clínicas y hemodinámicas entre sobrevivientes y no sobrevivientes.

\section{Métodos}

El protocolo fue aprobado por el Comité Local de Ética de la institución. Se realizó un estudio observacional, transversal y comparativo en neonatos atendidos en la UCIN de un hospital pediátrico de tercer nivel de atención en un periodo de 2 años (2017-2018). Se incluyeron neonatos a quienes, a solicitud del médico tratante, se les realizó EfN por un neonatólogo capacitado.

Las causas de la valoración se clasificaron en cinco grupos: a) persistencia de conducto arterioso (PCA); b) hipertensión arterial pulmonar aguda (HAPa); c) estado de choque; d) hipertensión arterial pulmonar crónica/ displasia broncopulmonar (HAPc); e) sospecha de trombos o vegetaciones.

Los estudios ecocardiográficos se realizaron con un equipo de ultrasonido Acuson $\times 300 \AA$ (Siemens Healthcare, Múnich, Alemania) con un transductor neonatal de $9 \mathrm{MHz}$ sincronizado con electrocardiograma. La toma de signos vitales que se utilizó para el análisis fue realizada al momento del estudio a través del monitor del paciente. Se establecieron dos protocolos para la evaluación ecocardiográfica:

- Completo o rutinario: se adquirieron imágenes en los seis ejes principales (subcostal, paraesternal largo, eje corto a nivel de los grandes vasos, apical de cuatro y cinco cámaras, y de ventrículo derecho de tres cámaras) en los modos bidimensional, Doppler color, Doppler pulsado y continuo, y M. 
- Por urgencia (en situaciones de inestabilidad hemodinámica con labilidad a cambios o con ventilación de alta frecuencia): se limitó a los ejes necesarios para realizar las mediciones hemodinámicas pertinentes de acuerdo con la patología principal.

La evaluación hemodinámica del ventrículo derecho (VD) se basó en las siguientes mediciones:

- Gasto cardiaco del $\mathrm{VD}^{8}$, medido en $\mathrm{ml} / \mathrm{kg} / \mathrm{min}$ [área de la válvula pulmonar $\times$ (integral del tiempo/velocidad) $\times$ frecuencia cardiaca], considerado como anormal cuando es menor que una desviación estándar (DE) de la media, dependiendo de si es prematuro o a término y si está durante o posterior al periodo de transición.

- TAPSE (excursión sistólica del anillo tricuspídeo de la base al ápex), medido en $\mathrm{mm}$, considerando como afectado su índice cuando es $<2$ DE según la edad gestacional ${ }^{9}$.

- Cambio de área fraccional del VD, calculado en modo bidimensional en cuatro cámaras o en tres cámaras tomando una imagen en telediástole y otra en telesístole con la fórmula ${ }^{10}$ [área telediastólica - área telesistólica / área tele diastólica $\times$ 100], expresado en porcentaje, considerando un valor $<35 \%$ como anormal.

De acuerdo con las recomendaciones del Neonatologist Performed Echocardiography (NPE) ${ }^{11}$, la evaluación de la poscarga del VD (hipertensión arterial pulmonar) se basó en las siguientes mediciones:

- Presión sistólica del VD: con la ecuación de Bernoulli simplificada, midiendo la velocidad pico de la regurgitación tricuspídea (en caso de existir espectro continuo del jet regurgitante en modo Doppler continuo) en $\mathrm{mmHg}$.

- Presión media de la arteria pulmonar: con la ecuación de Bernoulli simplificada, midiendo la velocidad pico de la insuficiencia pulmonar (en caso de existir en Doppler continuo) en $\mathrm{mmHg}$.

- Configuración del septo interventricular: considerando un septo plano como más del $50 \%$ de la presión del ventrículo izquierdo (VI) y un movimiento septal paradójico como presión suprasistémica.

- Cortocircuito bidireccional (derecha a izquierda en sístole) o de derecha a izquierda a través del foramen oval y el conducto arterioso.

- Intervalos sistólicos del VD: midiendo el tiempo transcurrido desde el inicio de la eyección de la válvula pulmonar hasta el punto máximo de aceleración, así como el tiempo transcurrido desde el inicio hasta el final de la eyección de la válvula pulmonar. Se considera un tiempo de aceleración corto (inversamente proporcional a las resistencias vasculares pulmonares) $<1 \mathrm{DE}$, dependiendo del peso y de la edad posnatal, indizándolo por el tiempo de eyección y expresándolo como índice de resistencias vasculares pulmonares ${ }^{12}$. La evaluación hemodinámica del VI se basó en las siguientes mediciones:

- Fracción de expulsión del VI obtenida por el método de Simpson o por planimetría, registrado en porcentaje $\mathrm{p}^{13,14}$.

- Fracción de acortamiento del VI expresada en porcentaje ${ }^{15}$.

- Gasto cardiaco del VI: con el mismo principio del gasto del VD, pero utilizando el área de la válvula aórtica ${ }^{8}$.

La evaluación del PCA se basó en las mediciones de acuerdo con las recomendaciones del NPE ${ }^{16}$, con el objetivo de ajustar el manejo farmacológico.

En los pacientes con PCA, imágenes con sospecha de cardiopatía congénita o trombos o vegetaciones, se realizó interconsulta con el servicio de cardiología pediátrica para corroborar el diagnóstico.

Los resultados se registraron en una base de datos actualizada diariamente con los registros del expediente clínico reportados en las evaluaciones de los médicos tratantes. Por cuestiones éticas, el operador del estudio ecocardiográfico reportó los resultados emitiendo recomendaciones terapéuticas, las cuales fueron integradas según el criterio del neonatólogo responsable. Se identificaron los pacientes no sobrevivientes con el expediente clínico y por llamada telefónica (en aquellos casos que no asistieron a la consulta de seguimiento). El proceso de este estudio involucró al neonatólogo evaluador (con EfN), a dos personas independientes que recabaron la información y a un investigador independiente que realizó el análisis estadístico. Ninguno se encontraba involucrado con el tratamiento de los enfermos.

Después de realizar el ecocardiograma, el neonatólogo evaluador emitió recomendaciones para todos los casos en una nota en la que se asentaban los resultados de la EfN. Se consideró como cambio terapéutico la presencia de una indicación inmediata en la hoja de indicaciones en ese turno.

\section{Análisis estadístico}

Se realizó estadística descriptiva. Las variables cualitativas se expresaron en frecuencias y porcentajes. Para las cuantitativas, se realizaron pruebas de normalidad, y aquellas con distribución paramétrica se expresaron en promedios y DE, y las no paramétricas, en medianas y rangos intercuartílicos (p25-p75). 
Tabla 1. Comparación de los diagnósticos, las características clínicas y los parámetros ecocardiográficos entre sobrevivientes y no sobrevivientes

\begin{tabular}{|c|c|c|c|c|c|c|c|}
\hline & \multicolumn{2}{|c|}{ Total $(n=119)$} & \multicolumn{2}{|c|}{ Sobrevivientes ( $n=101$ ) } & \multicolumn{2}{|c|}{ No sobrevivientes ( $n=18$ ) } & \multirow[t]{2}{*}{ p } \\
\hline & Frecuencia & $\%$ & Frecuencia & $\%$ & Frecuencia & $\%$ & \\
\hline $\begin{array}{l}\text { Diagnósticos* } \\
\text { Prematuro } \\
\text { SDR en niño a término } \\
\text { Hernia diafragmática } \\
\text { Sepsis } \\
\text { Quirúrgico abdominal } \\
\text { Prematuro tardío } \\
\text { Deshidratación hipernatrémica } \\
\text { MAV } \\
\text { Quirúrgico de tórax } \\
\text { Neuroquirúrgico }\end{array}$ & $\begin{array}{l}48 \\
22 \\
14 \\
9 \\
9 \\
5 \\
4 \\
3 \\
3 \\
2\end{array}$ & $\begin{array}{l}40.3 \\
18.5 \\
11.8 \\
7.6 \\
7.6 \\
4.2 \\
3.4 \\
2.5 \\
2.5 \\
1.7\end{array}$ & $\begin{array}{l}39 \\
20 \\
10 \\
8 \\
9 \\
5 \\
4 \\
3 \\
2 \\
1\end{array}$ & $\begin{array}{l}38.6 \\
19.8 \\
9.9 \\
7.9 \\
8.9 \\
5.0 \\
4.0 \\
3.0 \\
2.0 \\
1.0\end{array}$ & $\begin{array}{l}9 \\
2 \\
4 \\
1 \\
0 \\
0 \\
0 \\
0 \\
1 \\
1\end{array}$ & $\begin{array}{l}50.0 \\
11.1 \\
22.2 \\
5.6 \\
0.0 \\
0.0 \\
0.0 \\
0.0 \\
5.6 \\
5.6\end{array}$ & 0.5 \\
\hline & Mediana & P25-P75 & Mediana & P25-P75 & Mediana & P25-P75 & \\
\hline $\begin{array}{l}\text { Parámetros clínicosł } \\
\text { Edad gestacional (semanas) } \\
\text { Peso (kg) } \\
\text { Edad corregida (semanas) } \\
\text { Presión arterial sistólica (mm Hg) } \\
\text { Presión arterial diastólica }(\mathrm{mm} \mathrm{Hg} \text { ) } \\
\text { Frecuencia cardiaca (min) }\end{array}$ & $\begin{array}{c}35 \\
2.2 \\
37 \\
68 \\
40 \\
154\end{array}$ & $\begin{array}{c}30-38 \\
1.39-2.9 \\
31-40 \\
56-79 \\
31-49 \\
152-167\end{array}$ & $\begin{array}{c}35 \\
2.1 \\
37 \\
68 \\
42 \\
147\end{array}$ & $\begin{array}{c}30-38 \\
1.36-2.9 \\
33-40 \\
57-80 \\
31-51 \\
137-163\end{array}$ & $\begin{array}{c}32.6 \\
2.12 \\
33 \\
49 \\
30 \\
151\end{array}$ & $\begin{array}{c}30-38 \\
0.93-3 \\
30-38 \\
44-61 \\
23-39 \\
147-170\end{array}$ & $\begin{array}{c}0.4 \\
0.33 \\
0.09 \\
0.001 \\
0.008 \\
0.3\end{array}$ \\
\hline $\begin{array}{l}\text { Parámetros ecocardiográficos† } \\
\text { GC derecho (ml/kg/min) } \\
\text { TAPSE (mm) } \\
\text { CAF (\%) } \\
\text { PSVD (mm Hg) } \\
\text { Tiempo de eyección pulmonar (ms) } \\
\text { Tiempo de aceleración de la pulmonar (ms) } \\
\text { IRVp (ecocardiograma) } \\
\text { GC izquierdo (ml/kg/min) } \\
\text { FEVI (modo M) } \\
\text { FA (modo M) } \\
\text { FEVI (Simpson) }\end{array}$ & $\begin{array}{c}206 \\
8.8 \\
44 \\
42 \\
178 \\
50 \\
3.4 \\
186 \\
68 \\
35 \\
66\end{array}$ & $\begin{array}{c}151-255 \\
7-10 \\
38-52 \\
33-54 \\
160-190 \\
40-67 \\
2.64-4.4 \\
148-236 \\
62-72 \\
32-39 \\
62-70\end{array}$ & $\begin{array}{c}182 \\
8.7 \\
46 \\
40 \\
181 \\
50 \\
3.4 \\
181 \\
67 \\
34 \\
65\end{array}$ & $\begin{array}{c}145-235 \\
7.1-9.8 \\
40-53.3 \\
28-45 \\
163-197 \\
40-70 \\
2.6-4.5 \\
146-217 \\
62-72 \\
31-37 \\
61-69\end{array}$ & $\begin{array}{c}139.5 \\
7.9 \\
39 \\
47 \\
172 \\
43 \\
3.9 \\
152.5 \\
63 \\
36.5 \\
63\end{array}$ & $\begin{array}{c}84-187 \\
6.5-8.4 \\
35.46 .8 \\
34-64 \\
152-210 \\
37-52 \\
3.28-4.9 \\
100-235 \\
55-75 \\
29-42 \\
60-66\end{array}$ & $\begin{array}{c}0.01 \\
0.01 \\
0.03 \\
0.1 \\
0.6 \\
0.02 \\
0.07 \\
0.1 \\
0.33 \\
0.4 \\
0.3\end{array}$ \\
\hline
\end{tabular}

CAF: cambio fraccional de área; FA: fracción de acortamiento; FEVI: fracción de expulsión del ventrículo izquierdo; GC: gasto cardiaco; IRVp: índice de resistencias vasculares pulmonares; MAV: malformaciones arteriovenosas; PSVD: presión sistólica del ventrículo derecho; SDR: síndrome de dificultad respiratoria;

TAPSE: excursión sistólica del anillo tricuspídeo.

*Prueba $\chi^{2}$.

'Prueba U de Mann Whitney.

Se realizó un análisis bivariado. Los grupos de comparación fueron los sobrevivientes y los no sobrevivientes, y se utilizó la prueba $U$ de Mann Whitney para calcular el valor de probabilidad. Un valor de $\mathrm{p}<0.05$ se consideró estadísticamente significativo. Se utilizó el paquete estadístico SPSS (Statistics for Windows, versión 21.0).

\section{Resultados}

Durante el bienio estudiado ingresaron 509 pacientes en la UCIN, de los cuales 119 fueron evaluados con EfN. La mediana de edad gestacional fue de 35 (30-38) semanas y $69(58 \%)$ fueron de sexo masculino. Del total, $77(64.7 \%)$ fueron prematuros y $18(15 \%)$ fallecieron (Tabla 1).

Se realizaron 269 estudios a 119 neonatos. Cada paciente recibió una mediana de 2 (1-12) evaluaciones ecocardiográficas, y 248 (92\%) correspondieron al protocolo completo. Se realizaron 20 estudios $(7 \%)$ con ventilación de alta frecuencia, 158 (59\%) bajo ventilación mecánica convencional, $27(10 \%)$ en apoyo con presión continua en la vía aérea y 38 (14\%) con apoyo de oxígeno.

Sobre las indicaciones de los estudios (Tabla 2), 101 (38\%) fueron por HAPa, 73 (27\%) por PCA, 51 (19\%) por estado de choque, 38 (14\%) por HAPc y $6(2 \%)$ por la sospecha de trombos o vegetaciones. 
Tabla 2. Indicaciones para la valoración hemodinámica y hallazgos incidentales

\begin{tabular}{|l|c|c|}
\hline Hallazgo & Frecuencia & Porcentaje \\
\hline $\begin{array}{l}\text { Indicación de la valoración }(\mathrm{n}=269) \\
\text { HAPa }\end{array}$ & 101 & \\
PCA* & 73 & 27 \\
Choque & 51 & 19 \\
HAPc & 38 & 14 \\
Búsqueda de trombos/vegetaciones & 6 & 2 \\
\hline Hallazgos incidentales ( $\mathrm{n}=51)$ & & \\
Catéter mal posicionado & 37 & 14 \\
Trombos/vegetaciones* & 7 & 3 \\
Cardiopatía congénita* & 5 & 2 \\
Embolia aéreo & 1 & 0.37 \\
Derrame pleural & 1 & 0.37 \\
\hline
\end{tabular}

HAPa: hipertensión arterial pulmonar aguda; HAPc: hipertensión arterial pulmonar crónica; PCA: persistencia del conducto arterioso.

*Confirmación del diagnóstico por el servicio de cardiología pediátrica.

Acerca de las recomendaciones y los cambios en el tratamiento emitidos (Figura 1), en el $48.5 \%$ de los casos de HAPa $(n=101)$ se realizaron ajustes en el inicio y cambio de vasodilatadores pulmonares, vasopresores e inotrópicos; en el $26 \%$ de los casos de PCA $(n=73)$ se decidió el cierre farmacológico; en el $58 \%$ de los casos de estado de choque $(n=51)$ la recomendación fue cambiarlo por inicio/cambio del tratamiento aminérgico, y en el $10 \%$, este fue descartado; en los casos de HAPc $(n=38)$, en el contexto de cardiopatía congénita y repercusión hemodinámica se ajustó la dosis del diurético en el $47 \%$; finalmente, en los seis casos de sospecha de trombos o vegetaciones, el $66 \%$ fueron descartados. Se recomendó no intervenir farmacológicamente en el 7\% de las consultas. En 53 consultas $(20 \%)$ se dio alguna recomendación sobre el manejo ventilatorio (incrementar o disminuir la presión media en la vía aérea, presión positiva al final de la espiración o progresión del modo ventilatorio).

Se reportaron hallazgos incidentales durante la valoración en el $19 \%$ de los pacientes $(n=51)$, entre los cuales el más frecuente fue la malposición del catéter central (14\%), por lo que se procedió a su retiro o recolocación, seguida de trombos o vegetaciones, cuya resolución consistió en trombólisis/antibióticos (Tabla 2).

Se realizó el análisis comparativo entre los grupo de sobrevivientes y no sobrevivientes. Se encontraron diferencias cuantitativas con valores desfavorables en los no sobrevivientes en las siguientes variables: presión arterial sistólica y diastólica sistémicas, gasto cardiaco derecho, TAPSE, cambio de área fraccional del
VD y tiempo de aceleración de la arteria pulmonar (Tabla 1).

\section{Discusión}

En este estudio, el análisis de la aplicación de EfN se realizó en la UCIN de un hospital pediátrico, en la cual el $65 \%$ de los pacientes eran prematuros. La mortalidad global de los sujetos de investigación fue del $15 \%$, secundaria a complicaciones por HAPa, PCA y estado de choque, lo que ubica a este estudio en el contexto de los centros hospitalarios pertenecientes a hospitales pediátricos sin unidad de tococirugía, que reciben neonatos con patologías perinatales y quirúrgicas. En México, este escenario es frecuente en las instituciones de salud pertenecientes al sector público.

Sobre la disposición de la evaluación ecocardiográfica, la mediana de estudios por paciente fue de dos, lo que demuestra el uso de esta herramienta de manera longitudinal durante el proceso de atención y no exclusivamente para el paciente con una cardiopatía congénita. Actualmente existen cerca de 50 neonatólogos con entrenamiento en EfN en Canadá, México y los Estados Unidos, que son miembros de la PanAmerican Hemodynamic Collaborative, asociación conformada por un grupo de académicos que promueven la educación en hemodinamia neonatal mediante conferencias, seminarios en línea y aprendizaje a través de simuladores, y que realizan investigación colaborativa ${ }^{17}$.

Los centros que ofrecen adiestramiento en EfN han publicado los resultados de sus estudios. En Toronto, El-Khuffash, et al. ${ }^{18}$ (512 ecocardiogramas en 199 neonatos por 4 años) y Papadhima, et al. ${ }^{19}$ (553 ecocardiogramas en 268 neonatos por 4 años y 8 meses) realizaron cambios justificados en la EfN en el $41 \%$ y el $48 \%$ de los neonatos, respectivamente. En España, Corredera, et al. ${ }^{20}$ (168 ecocardiogramas en 50 neonatos por 1 año) ajustaron el manejo farmacológico en el $48 \%$ de los nenonatos. En Calgary, Harabor y Soraisham ${ }^{21}$ (303 ecocardiogramas en 129 neonatos, 18 de meses seguimiento) cambiaron el tratamiento en el $45 \%$ de los pacientes. En todos estos centros, la evaluación del PCA representó más del $50 \%$ de los motivos de la EfN (centros perinatales y de concentración de manejo quirúrgico de PCA). En el presente estudio, la causa más frecuente fue la HAPa, en el $38 \%$ de los pacientes, y el tratamiento se modificó en el $45 \%$ de los casos. Este comportamiento se explica debido a que la UCIN concentra un mayor número de neonatos con necesidades quirúrgicas, o a que algunos 


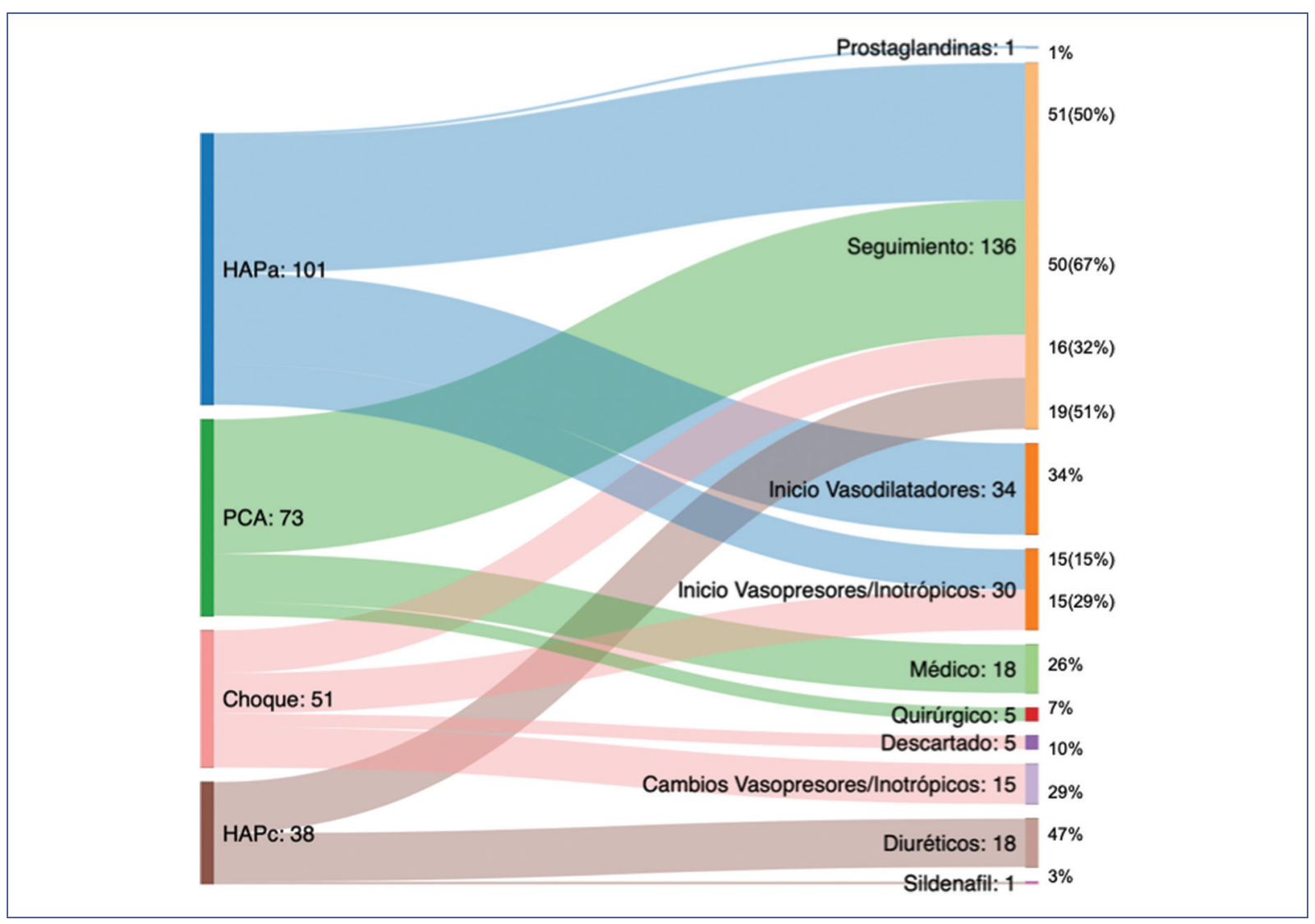

Figura 1. Motivos de la ecocardiografía funcional neonatal y recomendaciones. A la izquierda se dividen los motivos de solicitud de la evaluación con EfN. Cada franja de color se une en el extremo de la derecha con otra que significa el cambio realizado a partir de los resultados del estudio. El porcentaje expresado representa la proporción de la decisión terapéutica con respecto al motivo de estudio (por ejemplo, las prostaglandinas son el $1 \%$ de los cambios de la HAPa). Exclusivamente para la franja de seguimiento y el inicio de vasopresores/inotrópicos se decidió añadir el número total y el porcentaje correspondiente a cada patología. HAPa: hipertensión arterial pulmonar aguda; HAPc: hipertensión arterial pulmonar crónica; PCA: persistencia del conducto arterioso.

pacientes llegan de otros centros después del fracaso en las maniobras terapéuticas habituales.

Se conocen dos estudios en México sobre los resultados del programa de EfN, en los cuales un cardiólogo pediatra realizóla valoración. En el primero se evaluaron 15 neonatos (30 estudios) por inestabilidad hemodinámica y se justificó el cambio del manejo en 11 pacientes (73\%); a las 24 horas fueron reevaluados y 10 de ellos presentaron cambios ${ }^{22}$. En el segundo, en un centro privado, se analizaron 74 estudios en 37 neonatos, y se generaron cambios en el $70 \%$ de las ocasiones ${ }^{23}$.

La evaluación hemodinámica no solo permite escalar el tratamiento, sino también justificar una estrategia más conservadora y evitar los efectos adversos de los fármacos. Por ejemplo, El-Khuffash, et al. ${ }^{18}$ demostraron que en el $22 \%$ de sus evaluaciones se evitó una intervención o el inicio de fármacos. En cambio, en los pacientes de este trabajo lo anterior sucedió en el 7\%. Con esta misma intención, la evaluación por EfN permite la identificación de hallazgos incidentales. En este trabajo se identificaron en el 19\%, lo que concuerda con lo reportado por Smith, et al. ${ }^{24}$, quienes evidenciaron hallazgos incidentales en el $37 \%$ de las evaluaciones.

El análisis comparativo de las mediciones empleadas en la EfN para evaluar la función del VD en sobrevivientes y no sobrevivientes demostró un peor rendimiento en aquellos que fallecieron. Los valores de TAPSE y el cambio fraccional del área del VD fueron significativamente menores en los pacientes fallecidos. Al respecto, Malowitz, et al. ${ }^{25}$ encontraron resultados similares en neonatos > 35 semanas. Asimismo, Giesinger, et al ${ }^{26}$ demostraron datos consistentes con este índice en el contexto de la encefalopatía hipóxico-isquémica en tratamiento con hipotermia, y además 
coincide que el valor del cambio del área fraccional fue estadísticamente menor.

Otro índice ecocardiográfico es el tiempo de aceleración de la arteria pulmonar, cuya correlación es inversamente proporcional con las resistencias vasculares pulmonares al ser un subrogado de la poscarga del $V D$, lo que se demuestra en esta serie de pacientes al ser cuantitativamente menor en el grupo de sujetos fallecidos. Patel, et al. ${ }^{12}$ corroboraron que el valor de este índice es menor en aquellos que desarrollaron displasia broncopulmonar, en neonatos menores de 29 semanas. Por su parte, Levy, et al. ${ }^{27}$ cotejaron la consistencia de este fenómeno con el estudio de cateterismo cardiaco diagnóstico en 75 pacientes.

Por lo anterior, es creciente la necesidad de que los recursos humanos que atienden este tipo de enfermos se entrenen y adquieran las habilidades colegiadas para la adquisición y la interpretación de imágenes ecocardiográficas. En docencia, los grupos canadienses son los pioneros. En el centro hospitalario sede de este trabajo, los resultados se basan en la evaluación de un neonatólogo, quien es el único miembro del equipo del departamento de neonatología que realiza este tipo de valoraciones al momento.

De acuerdo con las recomendaciones internacionales, se puede proyectar el entrenamiento de otro miembro tras 2 años de práctica en el país de origen. En los reportes mencionados, los estudios se realizaron en Toronto por tres neonatólogos expertos o sus residentes entrenando bajo su programa ${ }^{18}$; en España, por dos neonatólogos del equipo ${ }^{20}$; en Calgary, todos fueron realizados por un solo operador ${ }^{21}$, como en el presente estudio.

La fortaleza de este estudio se basa en el análisis de la casuística y los resultados obtenidos por este programa. A la fecha, no hay reportes semejantes en México. Todas las inferencias y los análisis fueron realizados por personal médico capacitado por los programas avalados con formación en neonatología, y demuestran los efectos de la toma de decisiones.

Las debilidades del estudio radican en la naturaleza de la metodología utilizada, es decir, la observación del fenómeno desde el punto de vista transversal, lo que no permite el ajuste de variables confusoras, y la presencia de sesgo de mala clasificación en algunas variables.

En este estudio, la EfN se realizó en el $23.4 \%$ de los ingresos y el motivo principal fue la hipertensión pulmonar. Las mediciones ecocardiográficas de la disminución de la función sistólica y el aumento de la poscarga del VD se relacionaron con las muertes. En la mitad de las consultas se emitieron recomendaciones de ajuste terapéutico.

\section{Responsabilidades éticas}

Protección de personas y animales. Los autores declaran que para esta investigación no se han realizado experimentos en seres humanos ni en animales.

Confidencialidad de los datos. Los autores declaran que han seguido los protocolos de su centro de trabajo sobre la publicación de datos de pacientes.

Derecho a la privacidad y consentimiento informado. Los autores han obtenido el consentimiento informado de los pacientes y/o sujetos referidos en el artículo. Este documento obra en poder del autor de correspondencia.

\section{Conflicto de intereses}

Los autores declaran no tener ningún conflicto de intereses.

\section{Financiamiento}

Ninguno.

\section{Agradecimientos}

Al Dr. José Alberto García Aranda, la Mesa Directiva y el Patronato del Hospital Infantil de México Federico Gómez, por su apoyo para el programa. A Patrick J. McNamara y el Colaborativo Panamericano de Hemodinamia Neonatal, por la generosa capacitación.

\section{Bibliografía}

1. Harrison W, Goodman D. Epidemiologic trends in neonatal intensive care, 2007-2012. JAMA Pediatr. 2015;169:855-62.

2. Miller LE, Stoller JZ, Fraga MV. Point-of-care ultrasound in the neonatal ICU. Curr Opin Pediatr. 2020;32:216-27.

3. Weiss SL, Peters MJ, Alhazzani W, Agus MSD, Flori HR, Inwald DP, et al. Surviving sepsis campaign international guidelines for the management of septic shock and sepsis-associated organ dysfunction in children. Intensive Care Med. 2020;46:S10-67.

4. Mertens L, Seri I, Marek J, Arlettaz R, Barker P, McNamara P, et al. Targeted neonatal echocardiography in the neonatal intensive care unit: practice guidelines and recommendations for training. Eur J Echocardiogr. 2011;12:715-36.

5. de Boode WP, Singh Y, Gupta S, Austin T, Bohlin K, Dempsey E, et al Recommendations for neonatologists performed echocardiography in Europe: consensus statement endorsed by European Society for Paediatric Research (ESPR) and European Society for Neonatology (ESN). Pediatr Res. 2016;80:465-71.

6. Wyllie J. Neonatal echocardiography. Semin Fetal Neonatal Med. 2015;20:173-80.

7. Hebert A, Lavoie PM, Giesinger RE, Ting JY, Finan E, Singh Y, et al. Evolution of training guidelines for echocardiography performed by the neonatologist: toward hemodynamic consultation. J Am Soc Echocardiogr. 2019;32:785-90. 
8. de Boode WP, van der Lee R, Horsberg Eriksen B, Nestaas E, Dempsey $E$, Singh $Y$, et al. The role of neonatologist performed echocardiography in the assessment and management of neonatal shock. Pediatr Res. 2018;84:57-67.

9. Koestenberger M, Nagel B, Ravekes W, Urlesberger B, Raith W, Avian A, et al. Systolic right ventricular function in preterm and term neonates: reference values of the tricuspid annular plane systolic excursion (TAPSE) in 258 patients and calculation of Z-score values. Neonatology. 2011; $100: 85-92$

10. Jain A, Mohamed A, El-Khuffash A, Connelly KA, Dallaire F, Jankov RP et al. A comprehensive echocardiographic protocol for assessing neonatal right ventricular dimensions and function in the transitional period: normative data and z scores. J Am Soc Echocardiogr. 2014;27:1293-304.

11. de Boode WP, Singh Y, Molnar Z, Schubert U, Savoia M, Sehgal A, et al Application of neonatologist performed echocardiography in the assessment and management of persistent pulmonary hypertension of the newborn. Pediatr Res. 2018;84:68-77.

12. Patel MD, Breatnach CR, James AT, Choudhry S, McNamara PJ, Jain A, et al. Echocardiographic assessment of right ventricular afterload in preterm infants: maturational patterns of pulmonary artery acceleration time over the first year of age and implications for pulmonary hypertension. $J$ Am Soc Echocardiogr. 2019;32:884-94.

13. Jain A, El-Khuffash AF, Kuipers BCW, Mohamed A, Connelly KA, McNamara $\mathrm{PJ}$, et al. Left ventricular function in healthy term neonates during the transitional period. J Pediatr. 2017;182:197-203.

14. James AT, Corcoran JD, Jain A, McNamara PJ, Mertens L, Franklin O, et al. Assessment of myocardial performance in preterm infants less than 29 weeks gestation during the transitional period. Early Hum Dev. 2014;90:829-35.

15. Walther FJ, Siassi B, King J, Wu PY. Echocardiographic measurements in normal preterm and term neonates. Acta Paediatr Scand. 1986;75:563-8.

16. van Laere D, van Overmeire B, Gupta S, El-Khuffash A, Savoia M, McNamara PJ, et al. Application of NPE in the assessment of a patent ductus arteriosus. Pediatr Res. 2018;84:46-56.

17. Giesinger RE, Stanford AH, Rios DR, Bhombal S, Fraga MV, Levy VY, et al. Targeted neonatal echocardiography in the United States of America: the contemporary perspective and challenges to implementation. Pediatr Res. 2019;85:919-21.
18. El-Khuffash A, Herbozo C, Jain A, Lapointe A, McNamara PJ. Targeted neonatal echocardiography ( $\mathrm{TnECHO}$ ) service in a Canadian neonatal intensive care unit: a 4-year experience. J Perinatol. 2013; 33:687-90.

19. Papadhima I, Louis D, Purna J, Deshpande P, Diambomba Y, Lee S, et al. Targeted neonatal echocardiography (TNE) consult service in a large tertiary perinatal center in Canada. J Perinatol. 2018; 38:1039-45.

20. Corredera A, Rodriguez MJ, Arevalo P, Llorente B, Moro M, Arruza L. [Functional echocardiography in neonatal intensive care: 1-year experience in a unit in Spain]. An Pediatr (Barc). 2014;81:167-73.

21. Harabor A, Soraisham AS. Utility of targeted neonatal echocardiography in the management of neonatal illness. J Ultrasound Med. 2015; 34:1259-63.

22. Hernandez-Benitez R, Becerra-Becerra R. Functional echocardiography in the neonatal intensive care unit; experience in a tertiary level hospital. Bol Med Hosp Infant Mex. 2016;73:325-30.

23. Acevedo-Olguin AL, Hernandez-Benitez R, Iglesias-Leboreiro J, Bernardez-Zapata I, Gerardo-Del Hoyo MN, Rendon-Macias ME. Functional echocardiography as a tool for the dynamic treatment evaluation in critically ill neonates. Arch Cardiol Mex. 2019;89:393-98.

24. Smith A, Breatnach CR, James AT, Franklin O, El-Khuffash A. Incidental findings on routine targeted neonatal echocardiography performed in preterm neonates younger than 29 weeks' gestation. J Ultrasound Med. 2018:37:843-9.

25. Malowitz JR, Forsha DE, Smith PB, Cotten CM, Barker PC, Tatum GH. Right ventricular echocardiographic indices predict poor outcomes in infants with persistent pulmonary hypertension of the newborn. Eur Heart J Cardiovasc Imaging. 2015;16:1224-31.

26. Giesinger RE, El Shahed Al, Castaldo MP, Breatnach CR, Chau V, Whyte $\mathrm{HE}$, et al. Impaired right ventricular performance is associated with adverse outcomes after hypoxic-ischemic encephalopathy. Am J Respir Crit Care Med. 2019;200:1294-305.

27. Levy PT, Patel MD, Groh G, Choudhry S, Murphy J, Holland MR, et al. Pulmonary artery acceleration time provides a reliable estimate of invasive pulmonary hemodynamics in children. J Am Soc Echocardiogr. 2016;29:1056-65. 